\title{
NOTA SOBRE LA PRESENCIA DE NIEREMBERGIA ANGUSTIFOLIA (SOLANACEAE) EN JALISCO, MÉXICO
}

\author{
Luz Adriana García Rubio, Ofelia Vargas y Mollie Harker
}

Departamento de Botánica y Zoología, Centro Universitario de Ciencias Biológicas y Agropecuarias, Universidad de Guadalajara. Apartado postal 139 45100 Zapopan, Jalisco, México

\section{RESUMEN}

Se da a conocer la presencia de Nierembergia angustifolia en el estado de Jalisco, México. Se comentan los rasgos distintivos de esta especie y se discute su distribución geográfica conocida.

Palabras clave: Jalisco, México, Nierembergia, nuevo registro, Solanaceae.

\begin{abstract}
We report, for the first time, the presence of Nierembergia angustifolia in the state of Jalisco, Mexico. The distinctive characteristics of this species and an update of its known geographical distribution are discussed.
\end{abstract}

Key words: Jalisco, Mexico, new report, Nierembergia, Solanaceae.

\section{INTRODUCCIÓN}

Actualmente se realiza un trabajo florístico en el rancho Las Papas de Arriba, localizado en el municipio de Ojuelos de Jalisco, como parte de un estudio sobre la recuperación de suelos degradados en zonas semidesérticas. La revisión y determinación de ejemplares botánicos que provienen de esta área, nos revelaron la presencia de Nierembergia Ruiz \& Pavón, un género no registrado aún para la flora de Jalisco.

Nierembergia se distribuye en forma mayoritaria en América del Sur y agrupa cerca de 35 taxones (D’Arcy, 1978), 21 especies crecen en Argentina (Hunziker, 1979) y algunas otras en Colombia, Ecuador, Perú, Uruguay y Chile. El género no 
se registra de América Central. En Norteamérica se localiza una representante, Nierembergia angustifolia Kunth, que sólo se conoce de México. Con base en material colectado en Jalisco se presenta la descripción de esta planta.

Nierembergia angustifolia Kunth, Nov. Gen. et Sp. Pl. 3: 9. tab. 198. 1818. (Tipo: México, Hidalgo, Real del Monte, Humboldt \& Bonpland s. $n$.)

Hierba perenne, supina, de 9 a $20 \mathrm{~cm}$ de alto, ramificada en la base, tallos cilíndricos, angostos, de $1 \mathrm{~mm}$ de diámetro, algunos dicótomos, glabros o escasamente pilosos. Hojas simples, alternas, sésiles, de 1 a $2.5 \mathrm{~cm}$ de largo y de 1 a $2 \mathrm{~mm}$ de ancho, lámina linear-lanceolada, entera, aguda, rara vez redondeada o atenuada. Flores solitarias, axilares, pedicelo de 2-4 mm de largo; cáliz de 0.8-1.3 cm de largo, tubular-campanulado, 5-lobado, los lóbulos de 0.4 a $1.1 \mathrm{~cm}$ de largo y cerca de $1 \mathrm{~mm}$ de ancho, linear-lanceolados; corola blanca, hipocrateriforme, hasta de $2.1 \mathrm{~cm}$ de largo, el tubo muy angosto y exserto del cáliz, de 0.8 a $1.2 \mathrm{~cm}$ de largo, con tricomas glandulares, limbo acampanado que se extiende bruscamente en el ápice del tubo, de 0.9 a $1.2 \mathrm{~cm}$ de diámetro; estambres 5, connados en la base, insertos en la parte superior del tubo, desiguales, tres con los filamentos de $3 \mathrm{~mm}$ y los otros dos más cortos, de $2 \mathrm{~mm}$ de largo, anteras con las tecas iguales, de $1 \mathrm{~mm}$ de largo, amarillas; estilo de 1.2 a $1.5 \mathrm{~cm}$ de largo, simple, ensanchado hacia la parte apical, estigma capitado-laminar. Fruto una cápsula elipsoide, de 5-6 $\mathrm{mm}$ de largo y $3 \mathrm{~mm}$ de ancho, de color pardo claro, lisa; semillas numerosas, pequeñas, menores de $1 \mathrm{~mm}$, cuadrangulares, negras, foveoladas.

Las poblaciones de Nierembergia angustifolia en Jalisco no presentan diferencias morfológicas significativas con respecto a los ejemplares revisados de otras áreas del país. De los especímenes examinados, el material C. G. Pringle 4149 (MEXU), colectado en Morelia, es la planta más robusta y con el mayor tamaño conocido. Posee hojas hasta de $3 \mathrm{~mm}$ de ancho y los rasgos florales también muestran mayores dimensiones: los lóbulos del cáliz miden $1.5 \mathrm{~mm}$ de ancho, la corola alcanza los $2.5 \mathrm{~cm}$ de largo, el tubo floral y el limbo llegan a los $1.8 \mathrm{~cm}$ y $1.6 \mathrm{~cm}$ de largo respectivamente. En el resto de los ejemplares la variación es poca, se mantiene el porte pequeño y frágil de la planta, así como la forma y tamaño de las hojas; además son características constantes y diagnósticas, el tubo floral largo y angosto y el limbo abruptamente ensanchado.

Hunziker (1979) menciona que Nierembergia angustifolia y Bouchetia erecta DC. son similares en la estivación floral cocleada, el estigma laminar y el arreglo de los filamentos, pero difieren en que la última carece de un disco 
nectarífero. Ilustraciones del hábito y detalles de la estructura floral de $N$. angustifolia pueden consultarse en los trabajos de D’Arcy (1978: fig. 1-F y fig. 4-A, 4-B) y de Kunth (1818: lámina 198). En este último dibujo también se muestran rasgos del fruto, semilla y embrión.

Fenología: El período reproductivo de la especie comprende desde abril hasta diciembre.

Habitat: Nierembergia angustifolia crece en lugares con suelo húmedo o asociada a cuerpos de agua temporales y permanentes. Con frecuencia se localiza a orillas de charcos, ciénegas, arroyos y en los márgenes o alrededores de bordos, presas y lagunas. También se le encuentra dentro de cultivos de maíz, orillas de carreteras o en laderas de cerro en el pastizal y matorral xerófilo. La especie se desarrolla desde los $1820 \mathrm{~m}$ (Michoacán) hasta los $2500 \mathrm{~m}$ de altitud (Querétaro). En Jalisco, se colectó en el pastizal a la orilla de un jagüey, a 2260 m y en matorral xerófilo cerca de un arroyo, a $2000 \mathrm{~m}$.

Distribución: D’Arcy (1978) señala que Nierembergia angustifolia tiene una distribución amplia en México. En la actualidad se reconoce su presencia en 12 estados del país (Fig. 1), que incluyen a Durango, Zacatecas, Aguascalientes, Guanajuato, San Luis Potosí, Michoacán, Querétaro, Hidalgo (localidad tipo), Estado de México, Distrito Federal y Puebla (Rzedowski, 1954; D’Arcy, 1978; Rzedowski et al., 1996; García Regalado et al., 1999; Aguilar, 2001) y ahora se registra aquí para Jalisco. Los ejemplares depositados en los herbarios nacionales consultados (EBUM, IBUG, IEB, MEXU, SLPM) son pocos y las colectas no suman más de 20. Esto se debe quizás al tipo de habitat que requiere la planta, que consiste en la presencia de agua en zonas semi-áridas. Además, a esta planta se le ha referido como escasa en la mayoría de las colectas, aunque se menciona que es abundante de forma local en algunos sitios de Durango (González 3771), Michoacán (M. Díaz 55 y Díaz Barriga 7046) y Querétaro (Ocampo Acosta 945). Las poblaciones observadas en Jalisco no sobrepasan los 10 individuos.

Ejemplares revisados de Jalisco: municipio de Ojuelos de Jalisco, $2 \mathrm{~km}$ antes de llegar a Chinampas, 2000 m s.n.m., 29.V.1992, M. Cházaro-B. y M. NegreteA. 6946 (IBUG); municipio de Ojuelos de Jalisco, rancho Las Papas de Arriba, 2260 m s.n.m., 9.IX.2000, L. A. García-R. 298 (IBUG, IEB); ibid., 9.XII.2000, L. A. García-R. 487 (IBUG) e ibid., 9.VI.2000, L. A. García-R. 492 (IBUG). 


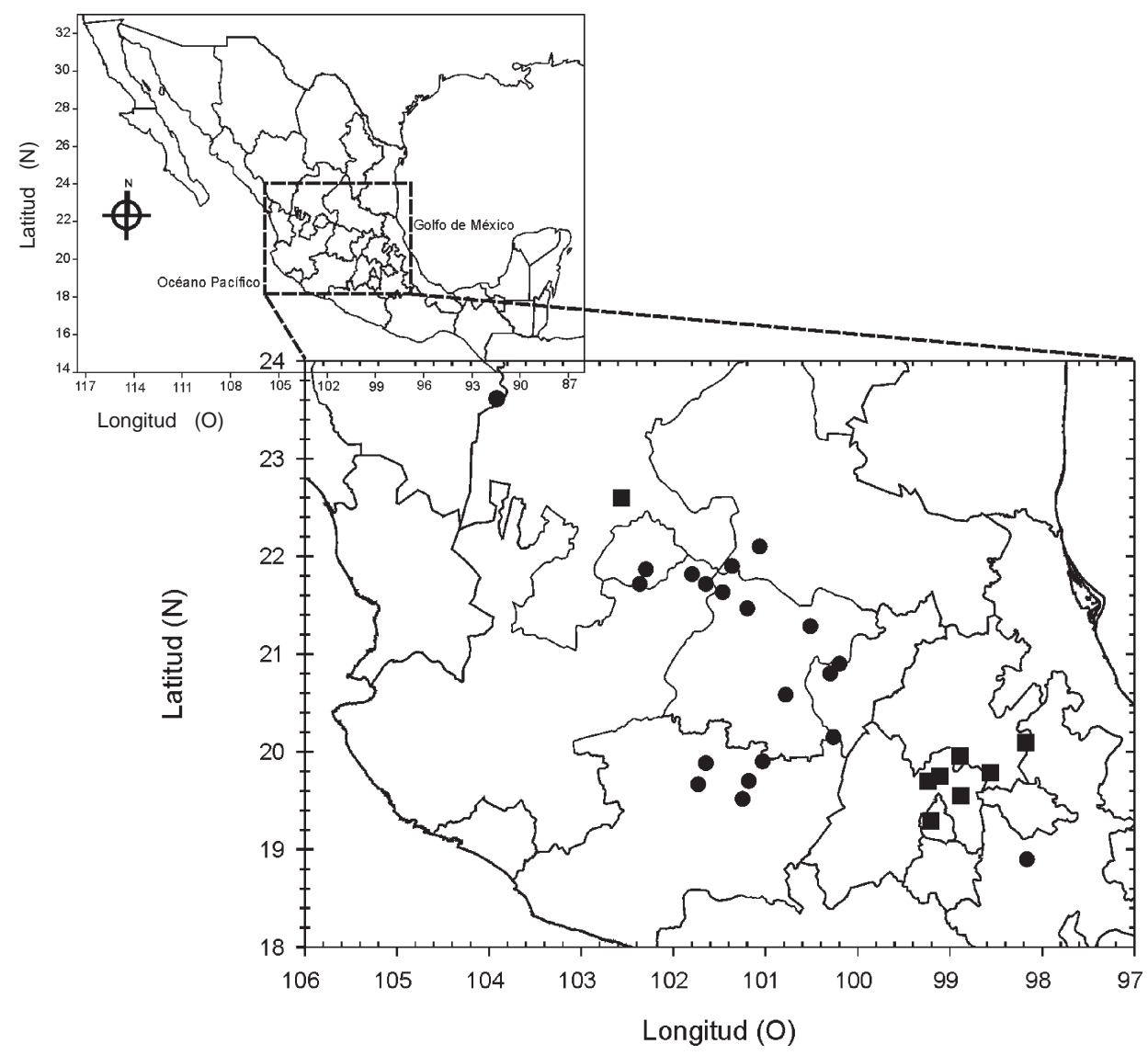

Figura 1. Mapa de la distribución conocida de Nierembergia angustifolia en México. (Simbología: - ejemplares consultados, $\square$ localidades de citas bibliográficas).

Ejemplares examinados de otros estados: Aguascalientes: municipio de Aguascalientes, Bordo Jacales, 1980 m s.n.m., 25.V.1987, M. E. Siqueiros 3646 (IEB); Rancho Aurora (17 km al E de Aguascalientes), 2000 m s.n.m., 23.IX.1996, M. de la Cerda 5899 (IEB). Durango: municipio de Súchil, $2 \mathrm{~km}$ al NE de Súchil, 13.X.1987, S. González 3771 (IEB, MEXU). Guanajuato: municipio de Ocampo, $3 \mathrm{~km}$ al $\mathrm{N}$ de Ocampo por la carretera a Ojuelos, $2300 \mathrm{~m}$ s.n.m., 10.IV.1990, J. Rzedowski 49407 (IEB, MEXU); municipio de San Felipe, $16 \mathrm{~km}$ al N de San Felipe por la carretera a Ocampo, 2250 m s.n.m., 13.VII.1987, J. Rzedowski 43715 (IEB); municipio de San Luis de La Paz, cerro El Kijay, 2200 m s.n.m., 10.IV.1989, E. Ventura y E. López 6625 (IEB); ibid., 1900 m s.n.m., 5.IV.1990, E. Ventura y 
E. López 7850 (IEB, MEXU). Michoacán: municipio de Álvaro Obregón, Potrero de las Cuatas, aprox. $3 \mathrm{~km}$ al E del poblado de La Presa, $1820 \mathrm{~m}$ s.n.m., 25.VIII.1984, M. Díaz 55 (IEB); municipio de Epitacio Huerta, cerca del poblado de La Paz, 14.X.1989, H. Díaz Barriga 6046 (IEB, MEXU); municipio de Erongarícuaro, Llano de Zinziro, 2400 m s.n.m., 2.VIII.1990, E. García y E Pérez 2902 (IEB, MEXU); municipio de Morelia, Ciénega cercana a Umécuaro, 2200 m s.n.m., 25.VI.1989, J. Rzedowski 48529 (IEB); municipio de Morelia, valley near Morelia, 21.VII.1892, C. G. Pringle 4149 (MEXU); municipio de Villa Jiménez, alrededores de Copándaro, 2000 m s.n.m., 24.IV.1988, J. Rzedowski 46490 (IEB, MEXU). Puebla: municipio de Puebla, laguna San Baltasar, 2121 m s.n.m., 20.IX.1906, G. Arsène 292 (MEXU). Querétaro: municipio de Colón, 3 km al N de Trigos, 2500 m s.n.m., 27.VII.1989, J. Rzedowski 48830 (IEB); municipio de El Marqués, cañada al E de la Presa El Carmen, 2050 m s.n.m., 24.IX.2000, G. Ocampo Acosta 945 (IEB). San Luis Potosí: municipio Villa de Arriaga, 3 miles E of Ojuelos, (ca. 50 miles W of San Luis Potosí), 11.IX.1978, W. G. D’Arcy 11871 (MEXU); municipio de San Luis Potosí, cerro Escalerillas, VI.1918, C. Conzatti 190 (MEXU); municipio de San Luis Potosí, Tenería, alrededor de la presa, 1900 m s.n.m., J. Rzedowski 3329 (SLPM).

\section{AGRADECIMIENTOS}

Agradecemos a Mónica Riojas López, quien dirige el proyecto en Ojuelos de Jalisco apoyado por CONACYT-SIMORELOS, con número 19980306-025, por su invitación a participar en los aspectos florísticos del estudio. La familia de Fernando Torres Romo prestó su casa y rancho para el trabajo de campo. Se reconoce la ayuda de Pablo Carrillo, Leticia Torres, Juan Antonio Reyes Agüero, Jorge Alberto Pérez de la Rosa y de los curadores de los herbarios citados. Se agradecen las observaciones y comentarios hechos al escrito original por Aarón Rodríguez Contreras y los revisores anónimos. Luz Ma. González Villareal nos facilitó copias de la descripción original de Kunth y fotocopias del dibujo.

\section{LITERATURA CITADA}

Aguilar, R. 2001. Solanaceae. In: Rzedowski, G. C. de, J. Rzedowski y colaboradores. Flora fanerogámica del Valle de México. 2a. ed. Instituto de Ecología, A. C. y Comisión Nacional para el Conocimiento y Uso de la Biodiversidad. Pátzcuaro, Michoacán. pp. 648-674. 
D’Arcy, W. G. 1978. A preliminary synopsis of Salpiglossis and other Cestreae (Solanaceae). Ann. Missouri Bot. Gard. 65: 698-724.

García Regalado, G., O. González Carrillo, M. de la Cerda Lemus y Ma. E. Siqueiros Delgado. 1999. Listado florístico del estado de Aguascalientes. Scientiae Naturae 1: 5-51.

Hunziker, A. T. 1979. South American: a synoptic survey. In: Hawkes, J. G., R. N. Lester y A. D. Skelding (eds.). Solanaceae: The biology and taxonomy of the Solanaceae. Academic Press. Londres. pp. 49-85.

Rzedowski, J. 1954. Vegetación del Pedregal de San Ángel (Distrito Federal, México). An. Esc. Nac. Cien. Biol., Méx. 8(1-2): 59-129.

Rzedowski, J., G. Calderón de Rzedowski y R. Galván. 1996. Nota sobre la vegetación y la flora del noreste del estado de Guanajuato. Flora del Bajío y de Regiones Adyacentes. Fascículo Complementario XIV. Instituto de Ecología, A.C. Centro Regional del Bajío. Pátzcuaro, Mich. 22 pp. 